P108 (continued)

meatless once a week would be good for their health. Middle school students showed no significant change in any parameter measured, and the majority of all students reported no change in eating behaviors.

Conclusions and Implication: The campaign increased awareness among elementary students about "meatless meals" but had no positive effect on other knowledge or behavior for any students. Nutrition education on the value of plant-based eating must confront resistance to change from the dominant meat eating culture.

Funding: None

\section{P109 Explorative Research of Food Presentation on Instagram Among Young Adults}

Gitte Kloek, PhD, g.c.kloek@hhs.nl, The Hague University of Applied Sciences, 75 Johanna Westerdijkplein, The Hague, The Netherlands; Stefanie Salmon, PhD, The Hague University of Applied Sciences; Sanne de Vries, PhD

Objective: Many youg adults use social media platforms as part of their daily routine. In order to develop effective health promoting interventions, social media may be an important factor in understanding young adult health behaviors. This study explores the presentation of food items on Instagram among Dutch young adults.

Study Design, Setting, Participants: To select participants between 18 to 24 years old we used images tagged with the hashtag \#[age]year. If the image referred to the birthday of the user, the account was included in the study. In total 350 accounts were identified (50 accounts per age group). The first image depicting food items posted at least two days prior to the \#[age]year image, was used in the analysis.

Outcome Measures and Analysis: A qualitative content analytic approach was used to analyze the food images. The images were categorized on what kind of food items and how they were displayed.

Results: Of the 350 accounts $(n=234$ women, $n=96$ men), food images were found in 272 (78\%) accounts. $83 \%$ of the women and $63 \%$ of the men shared food images $\left(\mathrm{Chi}^{2}, \mathrm{p}<0.001\right)$. Most identified food groups: cookies/pastries (19\%), fruits/vegetables (14\%), soda/ lemonade $(12 \%)$, meat $(11 \%)$, coffee/tea $(10 \%)$, and fast food (10\%). Food items were displayed: zoomed in on the food (67\%), in context with people/taking a selfie $(20 \%)$ or in a non-specific situation (13\%).

Conclusions and Implications: Most young adults share images showing food items with their followers, women more often than men. Although it is unclear whether these images represent actual behavior, it is interesting to know what food-related messages young adults share with one another.

Funding: ZonMw (The Netherlands Organization for Health Research and Development)
P110 Formative Testing of New SNAP-Ed Social Marketing Campaign Messages Designed for Low-Income Louisiana Residents

Brent Walker, MS, RD, brent.walker@altarum.org, Altarum Institute, 4 Milk Street, 3rd Floor, Portland, ME 04101; Brenda Wolford, $M S, R D$, Altarum Institute; Diane Sasser, PhD, Louisiana State University Agricultural Center; Carla Verbois, BA

Objective: To develop and focus group test new social marketing messages designed for low-income Louisiana residents.

Theory, Prior Research, Rationale: USDA's Food and Nutrition Service (FNS) recommends using comprehensive social marketing campaigns to expand reach of SNAP-Ed messages. In 2014, LSU AgCenter designed a SNAP-Ed social marketing campaign based on FNS' core nutrition messages reaching 32 parishes through billboards and print materials. In 2015, Altarum Institute conducted process and outcome evaluations of the campaign and findings were used to inform the 2016 campaign.

Description: Target Audience was SNAP-eligible Louisianans. After reviewing evaluation results, considering the needs of Louisiana's SNAP-eligible population, and reassessing the goals of Louisiana SNAP-Ed, LSU AgCenter developed new messages for their FFY 2017 campaign. Altarum Institute was contracted to assist with the development and testing of these new messages.

Evaluation: Eight focus groups were conducted statewide to test new messages within the themes of family mealtime, breakfast, physical activity and food safety.

Conclusions and Implications: The family mealtime theme tested favorably among focus group participants while the breakfast theme was not as well received. Participants responded well to the physical activity theme and recognized its importance for maintaining health. Future use of the food safety theme may require supplemental information at the local level to better educate the target audience. Participants would like to see visual images incorporated into campaign materials that represent the diversity of their communities and for message dissemination to include social media and community outreach channels. Formative testing of new social marketing messages was effective and provided useful feedback for informing future iterations of the campaign.

Funding: Supplemental Nutrition Assistance Program Education

\section{P111 Focus Groups Inform Children's Perceptions of Physical Activity, Screen Time and Sugar-Sweetened Beverages}

Denise Benoit-Moctezuma, MPH, University of Maryland, College Park; Kavitha Sankavaram, PhD, University of Maryland, College Park; Mira Mehta,PhD, mmehta@umd. edu, University of Maryland, College Park, 0102, Skinner Building, 4300 Chapel Lane, College Park, MD 20742

Continued on page 567 